\title{
HERPES ZOSTER INFECTION PRESENTING AS COMPLETE THIRD CRANIAL NERVE PALSY IN A PATIENT WITH SYSTEMIC LUPUS ERITHEMATOSUS: CASE REPORT
}

Thaynah Pisani Lisboa1, *, Eduardo Florim Terra1, Taíssa Cerqueira dos Santos1, Melissa Adriana Torres González1, Pedro Serra de Candol1, Camila Pitasi Arguelhes1, Camila Souto Oliveira Elias1

1.Universidade do Estado do Rio de Janeiro, Rio de Janeiro (RJ), Brazil.

*Corresponding author: thaypisani@gmail.com

\section{BACKGROUND}

Systemic lupus erythematosus (SLE) is a chronic inflammatory autoimmune disease that affects multiple organs. Neurological symptoms due to disease activity are difficult to distinguish from other illness sometimes. Infectious diseases remain an important etiology to rule out in SLE patients with neurologic presentation. It Is an important step before initiating immunosuppression. Herpes zoster $(\mathrm{HZ})$ reactivation is a matter of concern in immunocompromised patients, including SLE ones. There is a lack of established risk factors for $\mathrm{HZ}$ reactivation in SLE across studies. High-dose glucocorticoid, other immunosuppressive therapies, increasing age, lymphopenia and comorbidities are some of them. Neurological complications during $\mathrm{HZ}$ infection comprise a wide range of symptoms that include cranial nerve palsies. However, $\mathrm{HZ}$ uncommonly results in isolated ophthalmoplegia. Here we describe a case of $\mathrm{HZ}$ reactivation with complete third nerve palsy in a SLE patient in a referral center in Rio de Janeiro.

\section{CASE REPORT}

A 26-year-old female patient diagnosed with SLE with serosal, hematologic and pronounced cutaneous articular involvement since 2010 presented a 3-day history of vesicular painful rash in her dorsal and left inframammary regions (T5 dermatome) associated with dysesthesia. Her past medical data reveals several hypersensitivity reactions to therapies previously instituted (hydroxychloroquine, mycophenolate mofetil, cyclosporine). Thus, she was just on chronic use of prednisone $20 \mathrm{mg} /$ day. Neurologic exam revealed partial ophthalmoparesis and a fixed unilateral mydriasis in the left eye consistent with third cranial nerve palsy. Laboratory tests revealed only leukopenia with lymphopenia (2260 and 361/ $\mu \mathrm{L}$, respectively). Contrasted magnetic resonance of the brain and orbit did not show relevant abnormalities. Ophthalmologic examination excluded chorioretinitis, optic neuropathy and retinal vasculitis. Cerebrospinal fluid analysis showed mild pleocytosis. Screening for syphilis, gram staining, HSV 1 and 2, EBV, CMV, HHV-6 and HHV-8 resulted negative. Polymerase chain reaction amplification of VZV DNA in CSF was positive. Antiviral therapy was promptly started with intravenous acyclovir during 14 days along with corticotherapy with improvement of skin lesions and clinical status. Four weeks after discharge, she presented with progressive recovery of nerve palsy, but still had mild ptosis.

\section{CONCLUSION}

Herpes zoster reactivation in SLE is prone to occur given the immunological imbalance observed in the disease besides the immunosuppressive therapy. Third nerve palsy due to $\mathrm{HZ}$ reactivation is an infrequent manifestation, but its recognition is imperative in SLE patients, since neurologic symptoms of this infection can mimic active neurologic disease in SLE. 